\title{
PINK1/Parkin-mediated mitophagy is activated in cisplatin nephrotoxicity to protect against kidney injury
}

\author{
Ying Wang ${ }^{1}$, Chengyuan Tang ${ }^{1}$, Juan Cai ${ }^{1}$, Guochun Chen ${ }^{1}$, Dongshan Zhang ${ }^{2}$, Zhuohua Zhang ${ }^{3}$ and Zheng Dong ${ }^{1,4}$
}

\begin{abstract}
Cisplatin is a widely used chemotherapeutic drug with notorious toxicity in the kidneys, which involves mitochondrial dysfunction and damage in renal tubular cells. Mitophagy is a form of selective autophagy that removes damaged or dysfunctional mitochondria to maintain cellular homeostasis. In this study, we have used mouse and cell models to examine the role and regulation of mitophagy in cisplatin nephrotoxicity. Cisplatin treatment was associated with the activation of autophagy and mitophagy. Rapamycin, a pharmacological inhibitor of mTOR, stimulated autophagy and mitophagy, and alleviated the development of cisplatin nephrotoxicity. PINK1 and Parkin were increased in kidney tissues during cisplatin treatment of mice. In PINK1 or Parkin gene knockout mouse models, both basal and cisplatininduced mitophagy in kidneys were defective. Compared with wild-type littermates, PINK1 and Parkin knockout mice showed more severe renal functional loss, tissue damage, and apoptosis during cisplatin treatment. The results suggest that PINK1/Parkin-mediated mitophagy is activated in cisplatin nephrotoxicity and has a protective role against kidney injury.
\end{abstract}

\section{Introduction}

Cisplatin is a widely used chemotherapy drug for the treatment of cancers of the testis, ovary, breast, lung, and other origins ${ }^{1,2}$. However, the use of cisplatin is limited by its side effects in normal tissues, especially it preferentially accumulates in the kidney to cause DNA damage, mitochondrial dysfunction, and various stress responses in renal tubular cells, leading to tubular cell injury, dysfunction, and even death ${ }^{3-6}$. The mechanism underlying cisplatin nephrotoxicity is multifactorial, involving multiple signaling pathways and molecules ${ }^{3-9}$. It is essential to understand these pathways to identify effective ways to alleviate cisplatin nephrotoxicity in chemotherapy.

\footnotetext{
Correspondence: Zheng Dong (zdong@csu.edu.cn) (zdong@augusta.edu) ${ }^{1}$ Department of Nephrology, The Second Xiangya Hospital at Central South University, Changsha, Hunan, China

2Department of Emergency Medicine, The Second Xiangya Hospital at Central South University, Changsha, Hunan, China

Full list of author information is available at the end of the article.

Edited by B. Zhivotovsky
}

Mitochondria, the "power plant" in cells, are the primary organelles that generate adenosine triphosphate (ATP) via oxidative phosphorylation. As such, mitochondria are critically important for the maintenance of cellular viability and activities. Damage and dysfunction of mitochondria have an important role in the pathogenesis of renal diseases, including acute kidney injury (AKI) induced by renal ischemia-reperfusion, sepsis, and nephrotoxins ${ }^{10-14}$. These conditions are associated with the pathological changes of mitochondrial structure and function in kidney tissues, especially in renal tubules. Mitochondrial dysfunction would then result in oxidative stress, inflammation, and cell death, inducing a rapid deterioration in renal function. This seems particular true for cisplatin-induced AKI or nephrotoxicity ${ }^{3-6}$.

Mitochondria-specific autophagy (mitophagy) is a fundamental process that removes excessive or damaged mitochondria selectively via autophagy, which contributes to mitochondrial quality control and cell survival ${ }^{15-17}$. As a selective form of autophagy, mitophagy makes use of the

\section{(c) The Author(s) 2018}

(c) (i) Open Access This article is licensed under a Creative Commons Attribution 4.0 International License, which permits use, sharing, adaptation, distribution and reproduction cc) in any medium or format, as long as you give appropriate credit to the original author(s) and the source, provide a link to the Creative Commons license, and indicate if changes were made. The images or other third party material in this article are included in the article's Creative Commons license, unless indicated otherwise in a credit line to the material. If material is not included in the article's Creative Commons license and your intended use is not permitted by statutory regulation or exceeds the permitted use, you will need to obtain permission directly from the copyright holder. To view a copy of this license, visit http://creativecommons.org/licenses/by/4.0/. 
core machinery of autophagy for the formation of autophagosomes and autolysosomes. However, mitophagy requires a special priming process to label the mitochondria that are destined to autophagic degradation. Several pathways of mitochondrial priming have been described for mitophagy ${ }^{15-17}$. In this regard, the PINK1/ Parkin pathway is recognized as the main pathway for mitophagy under cell stress ${ }^{15-17}$. Normally, PINK1 enters mitochondria to reach the mitochondrial inner membrane where it is processed by the intramembrane serine protease PARL for degradation. Interestingly, the import of PINK1 depends on mitochondrial membrane potential. And when mitochondria are depolarized in conditions such as cell stress, PINK1 accumulates on the mitochondrial outer membrane, where it recruits and phosphorylates Parkin, an E3 ubiquitin ligase. Upon phosphorylation, Parkin is activated to induce the ubiquitination of various mitochondrial outer membrane proteins $^{18,19}$. Ubiquitin-tagged outer membrane proteins are then recognized by $\mathrm{p} 62 / \mathrm{SQSTM} 1$ leading to encapsulation by autophagosomes and final degradation by autolysosomes ${ }^{20-22}$.

Mitophagy contributes critically to the pathogenesis of various diseases, such as neurodegenerative diseases, metabolic diseases, ischemia-reperfusion injury ${ }^{23-25}$. Our recent work demonstrated the activation and protective role of the PINK1/Parkin pathway of mitophagy in renal ischemia-reperfusion $^{26}$. Yuan and colleagues further suggested a protective role of the PINK1/Parkin pathway during cisplatin treatment of HK2 renal tubular cells ${ }^{27}$. However, the role and regulation of PINK1/Parkinmediated mitophagy in cisplatin nephrotoxicity remains largely unclear, requiring in vivo evidence to establish. Our current study was designed to investigate PINK1/ Parkin-mediated mitophagy in cisplatin nephrotoxicity using both cell culture and animal models, including PINK1 and Parkin knockout (KO) mice.

\section{Results}

\section{PINK1/Parkin-dependent mitophagy is induced during} cisplatin treatment in BUMPT cells

We initially examined the PINK1/Parkin-mediated pathway of mitophagy during cisplatin treatment of BUMPT cells. Cisplatin treatment led to time-dependent apoptosis as shown by cell and nuclear morphologies. Apoptosis was noticeable at $16 \mathrm{~h}$ and, at $24 \mathrm{~h}$, many cells showed cellular shrinkage and blebbing, and nuclear condensation and fragmentation (Fig. 1a). Quantification by counting these cells revealed that cisplatin-induced $43 \%$ apoptosis in BUMPT cells for $24 \mathrm{~h}$ (Fig. 1b). In immunoblot analysis (Fig. 1c), cisplatin-induced LC3I to LC3 II conversion or LC3 II accumulation, indicative of the activation of autophagy. These cells also showed a reduction of the mitochondrial membrane protein TIM23 (translocase of inner mitochondrial membrane 23) and TOM20 (translocase of outer mitochondrial membrane 20 homolog), suggesting that mitochondrial clearance by mitophagy. Moreover, the expression of PINK1 and Parkin was increased, suggesting activation of PINK1-Parkin pathway of the mitophagy. Quantification of the blots by densitometry further verified the protein changes starting from 12 to $16 \mathrm{~h}$ and reaching high levels at $24 \mathrm{~h}$ of cisplatin treatment (Fig. 1d). To directly observe mitophagy, we examined the formation of "mitophagosomes" by co-localizing mitochondria and autophagosomes. BUMPT cells were transfected with LC3-GFP plasmids to reveal autophagosomes and Mito-Tracker was used to label mitochondria. The cells were subjected to cisplatin treatment or incubated in normal culture medium as control. As shown in Fig. 1e, control cells had very few GFP-LC3 puncta indicating a low level of autophagy. In sharp contrast, more LC3-GFP puncta were observed in BUMPT cells following cisplatin treatment, indicating autophagosome formation. Importantly, in cisplatintreated cells, some LC3-GFP-labeled puncta/autophagosomes co-localized with Mito-Tracker-labeled mitochondria (Fig. 1e), indicating mitophagy. These results provided the evidence of the activation of autophagy and mitophagy during cisplatin treatment of BUMPT cells, which was accompanied by the development of apoptosis.

\section{Mitophagy is activated in proximal tubular cells in C57/BL6 mice}

In vivo, we initially identified the occurrence of autophagy/mitophagy during cisplatin treatment in C57/BL6 mice. C57BL/6 mice (male, 8-10 weeks old) were intraperitoneally injected with $30 \mathrm{mg} / \mathrm{kg}$ cisplatin or saline as control. Blood samples were harvested to measure serum creatinine and blood urea nitrogen (BUN) to indicate the decline of renal function, and kidney tissues were collected for immunoblot/histology/apoptosis analysis at 24, 48 , and $72 \mathrm{~h}$. BUN and serum creatinine showed moderate increases at 24 and $48 \mathrm{~h}$ after cisplatin injection, and reached to 170 and $2.31 \mathrm{mg} / \mathrm{dl}$ at $72 \mathrm{~h}$, respectively (Fig. 2a, b). Histological analysis following H\&E staining revealed that tubular injury began to appear at $24 \mathrm{~h}$ and became severe at $72 \mathrm{~h}$ reaching tubular damage score of 2.53 (Fig. 2c, d). TdT-mediated dUTP nick-end labeling (TUNEL) assay showed that a small number of apoptosis cells began to appear at $24 \mathrm{~h}$, and significant apoptosis was observed at $72 \mathrm{~h}$ (Fig. 2c-e). In immunoblot analysis, we detected the changes in two biochemical markers of autophagy, including the increase of LC3II and the dramatic decrease of p62 (Fig. 2f). Following cisplatin injection, the mitochondrial membrane protein TOM20 and TIM23, and the mitochondrial matrix protein HSP60 (Fig. 2f-h) were reduced, suggesting mitophagy activation. Moreover, cisplatin-induced increases in both PINK1 and 
A
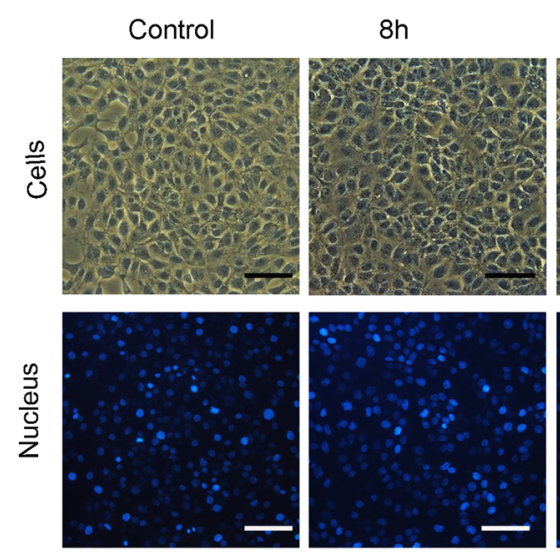

C

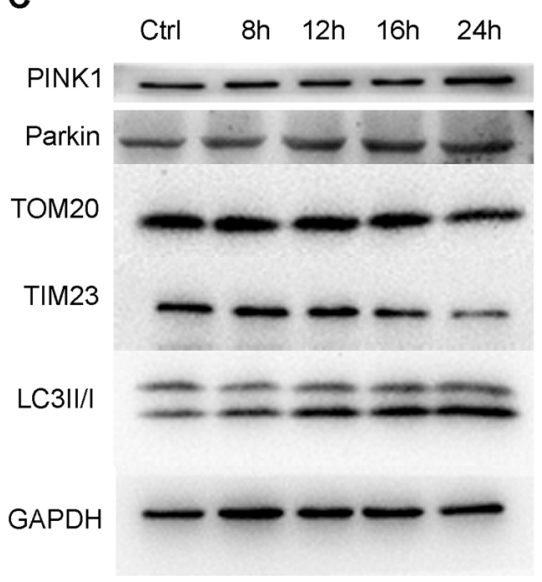

E
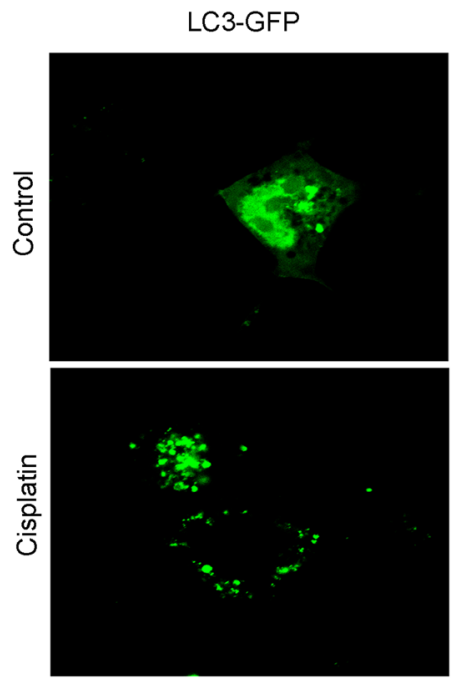

$8 \mathrm{~h}$
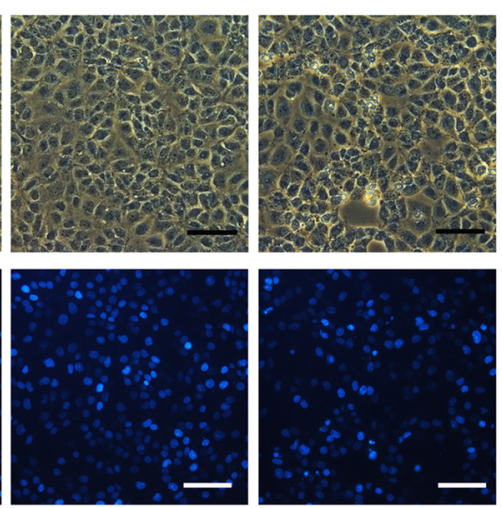

$16 \mathrm{~h}$
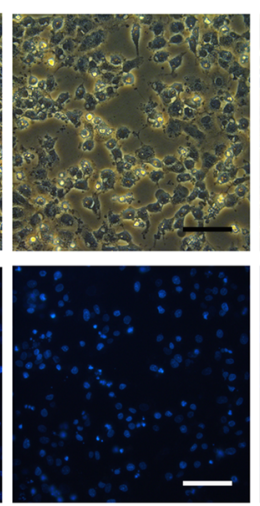

B

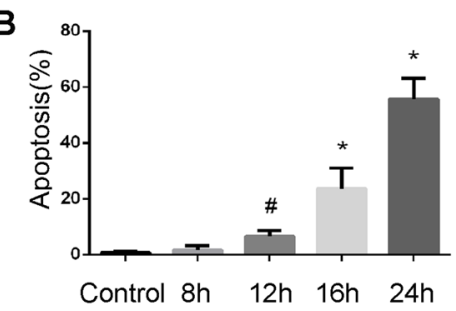

$-22 k D a$

- 16kDa

- 14kDa

$-37 \mathrm{kDa}$

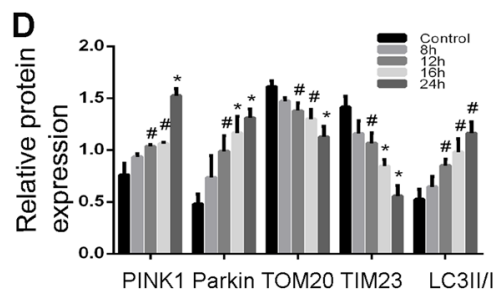

Mito-Tracker
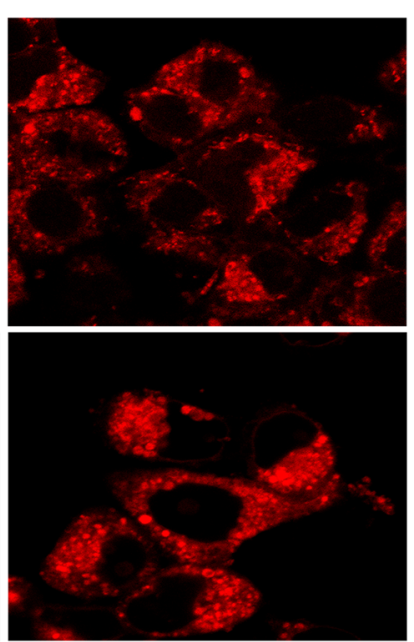

$24 \mathrm{~h}$
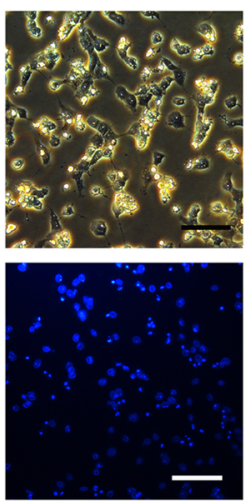
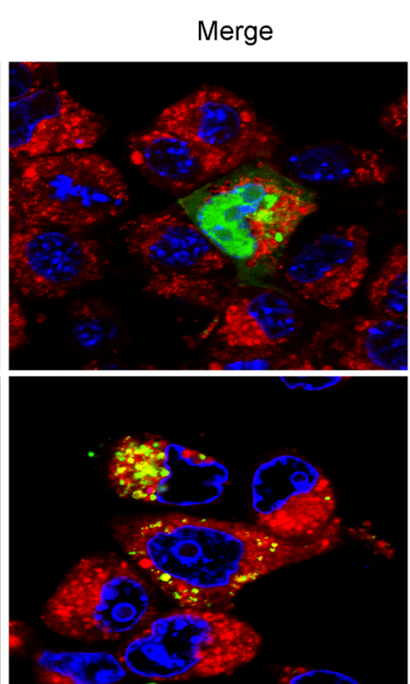

Fig. 1 PINK1/Parkin-mediated mitophagy is induced during cisplatin treatment in BUMPT cells. BUMPT cells were treated with $20 \mu M$ cisplatin for $0-24 \mathrm{~h}$. a Representative images of cellular and nuclear morphologies. After treatment, cells were stained with Hoechst33342 to examine by phase-contrast and fluorescence microscopy. Scale bar, $50 \mu \mathrm{m}$. b Apoptosis percentage. Apoptosis was evaluated to determine the cells with typical apoptotic morphology. c Whole cell lysate was collected for immunoblot analysis for PINK1, Parkin, TOM20, TIM23, LC3 II/I, and GAPDH (loading control). $\mathbf{d}$ Densitometry analysis of proteins signals on immunoblots The proteins signals were divided by GAPDH signal of the same samples to determine the ratios. e Co-localization of autophagosomes with mitochondria upon cisplatin treatment. BUMPT cells transiently transfected with LC3GFP were subjected to control or cisplatin treatment. Mitochondria in these cells were then labled with MitoTracker Red FM. The cells were examined by confocal microscopy to show the co-localiztion of autophagosomes (green LC3-GFP puncta) and mitochondria (red). Scale bar: $20 \mu \mathrm{m}$. Data in b and $\mathbf{d}$ are expressed as mean $\pm \mathrm{SD} . n=3 .{ }^{*} P<0.001,{ }^{\#} P<0.05$, significantly different from the control group 
A

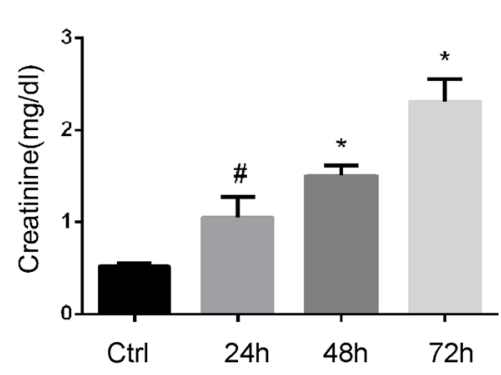

C
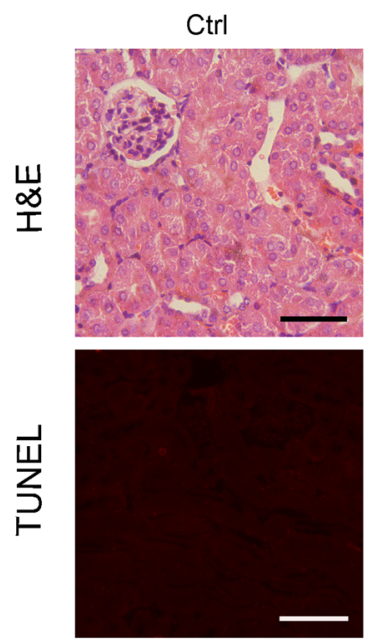

D

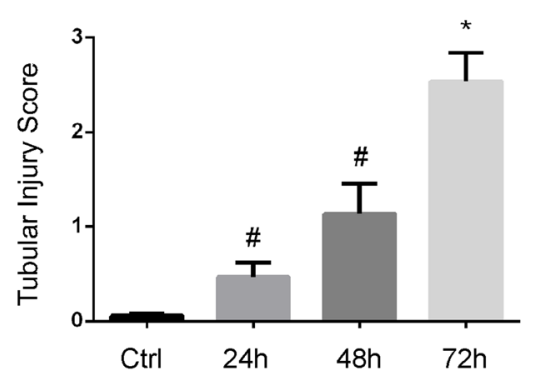

F

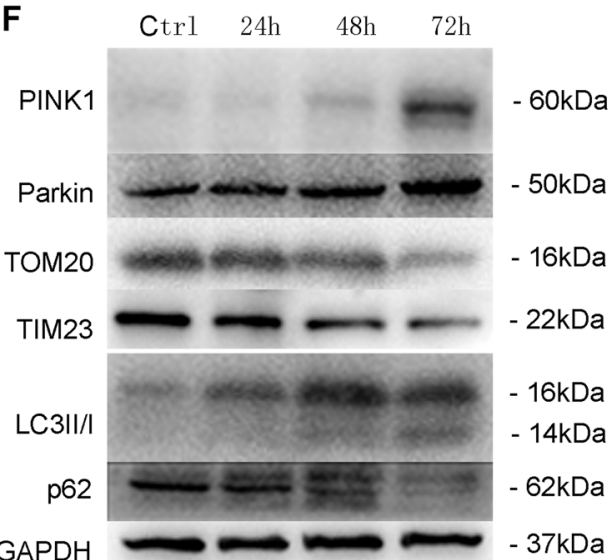

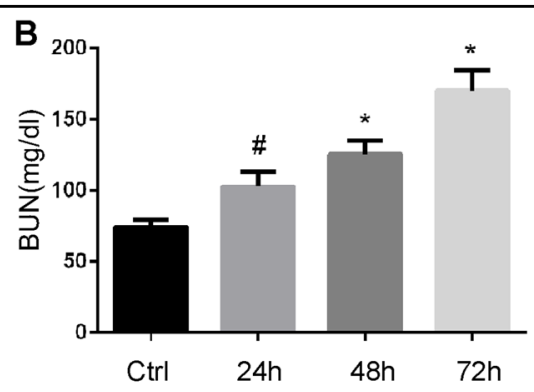

$48 \mathrm{~h}$
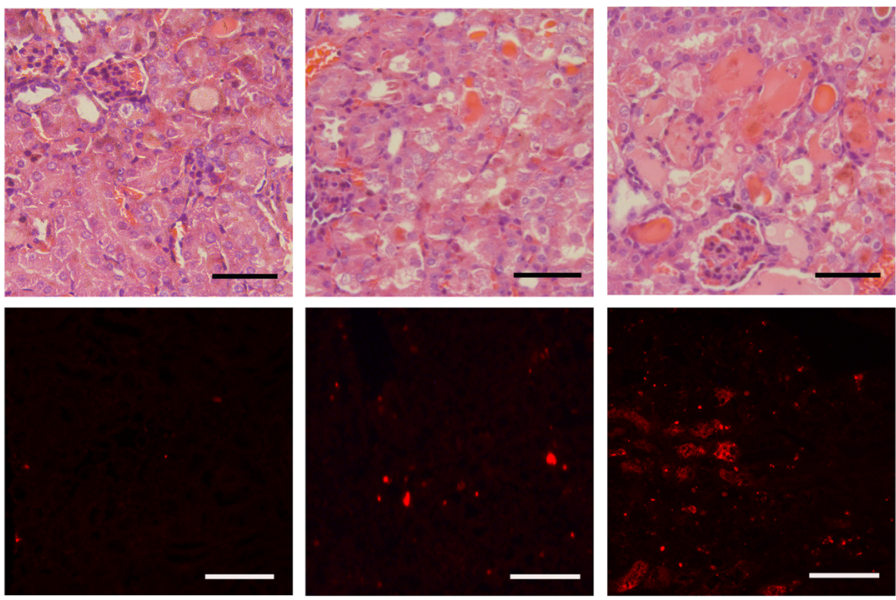

E

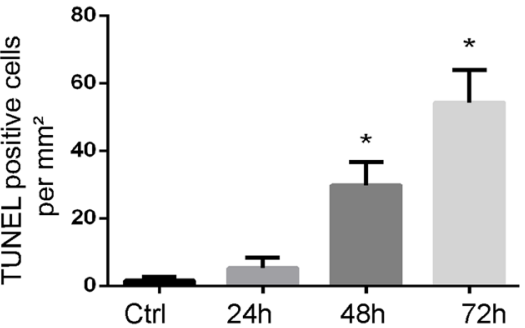

G

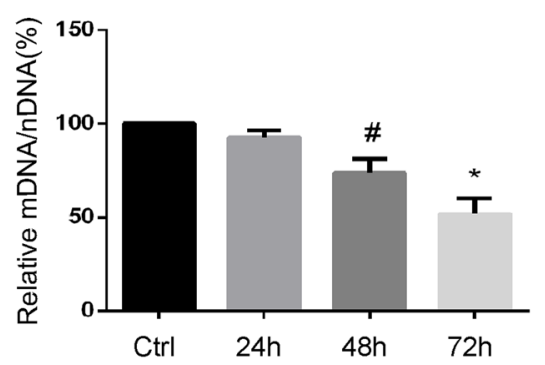

H

Ctrl $24 \mathrm{~h} \quad 48 \mathrm{~h} \quad 72 \mathrm{~h}$

HSP6O

$-60 \mathrm{kDa}$

GAPDH

$-37 \mathrm{kDa}$

Fig. 2 (See legend on next page.) 
(see figure on previous page)

Fig. 2 Mitophagy is induced in cisplatin-induced nephrotoxicity in mice. C57BL/6 mice (male, 8-10 weeks old) were injected with $30 \mathrm{mg} / \mathrm{kg}$ cisplatin or saline and divided into four groups for following treatments, respectively: (1) saline control; (2) cisplatin treatment for $24 \mathrm{~h}$; (3) cisplatin treatment for $48 \mathrm{~h}$; and (4) cisplatin treatment for $72 \mathrm{~h}$. a, b. Blood samples were collected for measurements of serum creatinine (a) and BUN (b). c Upper panel: Representative histology of kidney cortex shown by hematoxylin and eosin (H\&E) staining. Tubular injury was indicated by tubular dilation/flattening, loss of brush border, sloughing of cells into tubular lumen, formation of tubular casts, tubular degeneration, and vacuolization. Lower panel: Representative images of kidney cortex shown by TUNEL staining. Scale bar, $50 \mu \mathrm{M}$. $\mathbf{d}$ Tubular injury evaluated by counting the renal tubules with signs of injury. Tissue injury was scored by the percentage of damaged renal tubules (0, no damage; 1, $225 \% ; 2,25-50 \%$; 3, 50-75\%; and 4, >75\%). e Quantification of TUNEL-positive cells in cisplatin treatment for 24, 48, and $72 \mathrm{~h}$. f Relative mitochondrial DNA content (mtDNA/nDNA). g Immunoblot analysis of PINK1, Parkin, TOM20, TIM23, LC3II/I, p62, and GAPDH (loading control) in kidney tissues. $\mathbf{h}$ Immunoblot analysis of HSP60 and GAPDH (loading control). Data in $\mathbf{a}, \mathbf{b}$ and $\mathbf{d}$, $\mathbf{f}$ are expressed as mean $\pm \mathrm{SD}, n=3,{ }^{\sharp} P<0.05,{ }^{*} P<0.001$, significantly different from the control group

Parkin (Fig. 2g). mtDNA/nDNA ratio decreased in kidney tissues of cisplatin-treated mice (Fig. 2g), further indicating the clearance of mitochondria by mitophagy. When the mtDNA/nDNA ratio in control tissue was set as 1 , it decreased to 0.52 at the end of $72 \mathrm{~h}$ of cisplatin treatment (Fig. 2g). Together, these results indicate that the PINK1/ Parkin pathway of mitophagy is activated in kidney tissues during cisplatin nephrotoxicity in vivo.

\section{Rapamycin attenuates cisplatin nephrotoxicity in C57BL/6 mice}

To assess the role of mitophagy on cisplatin-induced AKI in C57BL/6 mice, we investigated the effect of rapamycin, a pharmacological inhibitor of mTOR that activates autophagy. Cisplatin-treated mice had $2.25 \mathrm{mg} /$ $\mathrm{dl}$ serum creatinine and $171.3 \mathrm{mg} / \mathrm{dl}$ BUN, which were reduced to 1.50 and $133.6 \mathrm{mg} / \mathrm{dl}$, respectively by rapamycin (Fig. 3a, b). Consistently, histological analysis revealed that rapamycin treatment alleviated renal tubular damage during cisplatin treatment (Fig. 3c). The tubular damage scores were 2.5 and 1.6, respectively for the cisplatin-only and the cisplatin + rapamycin groups (Fig. 3d). TUNEL assay revealed that there was significantly less tubular cell apoptosis in mice with cisplatin + rapamycin compared with those treated with cisplatinonly. To monitor mitophagy, we detected the expression of TOM20, TIM23, and HSP60 by immunoblot analysis. Cisplatin induced decreases in TOM20, TIM23, and HSP60 in renal cortical tissues, which were augmented by rapamycin (Fig. 3g-i). Rapamycin also further increased LC3-II and decreased p62 during cisplatin treatment (Fig. 3g, h). Moreover, rapamycin induced increases in both PINK1 and Parkin (Fig. 3j). Collectively, these results verified the enhancement of mitophagy and autophagy by rapamycin, which was associated with its protective effect against cisplatin nephrotoxicity.

\section{Mitophagy in cisplatin nephrotoxicity is attenuated in Parkin- or PINK1-KO mice}

To determine the role of PINK1/Parkin pathway of mitophagy in cisplatin-induced nephrotoxicity, we tested
Parkin- or PINK1-KO mouse models. We first verified Parkin deficiency in kidney tissues of Parkin-KO mice (Fig. 4a, b). We then examined mitophagy during cisplatin treatment of wild-type (WT) and Parkin-KO mice. As shown in Fig. 4c-f, cisplatin-induced obvious decreases in TIM23, TOM20, and HSP60 in WT kidney tissues, but the decreases were significantly less in Parkin-KO mice, suggesting the involvement of PINK1/Parkin pathway in mitophagy in cisplatin nephrotoxicity.

We further examined PINK1-KO mouse model. PINK1 deficiency in these mice was confirmed by genotyping and immunoblotting (Fig. 5a, b). We then compared the decreases of mitochondrial proteins TOM20, TIM23, and HSP60 induced by cisplatin in PINK1-KO and WT mice. As shown in Fig. $5 \mathrm{c}-\mathrm{e}$, cisplatin treatment reduced TOM20, TIM23, and HSP60 in WT kidney tissues, and the reduction was partially suppressed in PINK1-KO mice. Densitometry analysis further supported this conclusion (Fig. $5 d-f$ ). We further analyzed the recruitment of Parkin to mitochondria during mitophagy. To this end, mitochondria were isolated from renal cortex of cisplatintreated or control mice, and then subjected to immunoblot analysis. As shown in Fig. 5g, h, cisplatin induced an evident Parkin recruitment to mitochondrial fraction in WT mouse kidneys, which was largely attenuated in PINK1-deficient mice. The results suggested that PINK1 contributed to mitophagy in cisplatin nephrotoxicity.

\section{Cisplatin nephrotoxicity is aggravated in Parkin- or PINK1- KO mice}

Under unchallenged conditions, serum creatinine, and BUN were at low levels in both Parkin-KO mice and their WT littermates, indicating that renal function was normal in these mice (Fig. 6a, b). $72 \mathrm{~h}$ after cisplatin injection, WT mice developed acute renal failure, with serum creatinine increased to $2.12 \mathrm{mg} / \mathrm{dl}$. In the same experiments, Parkin$\mathrm{KO}$ mice had more severe loss of renal function, with serum creatinine of $3.06 \mathrm{mg} / \mathrm{dl}$ (Fig. 6a). However, there were showed similar levels of BUN in these two groups (Fig. 6b). In histological analysis following HE staining, Parkin-KO mice showed more renal tubule damage than 
A

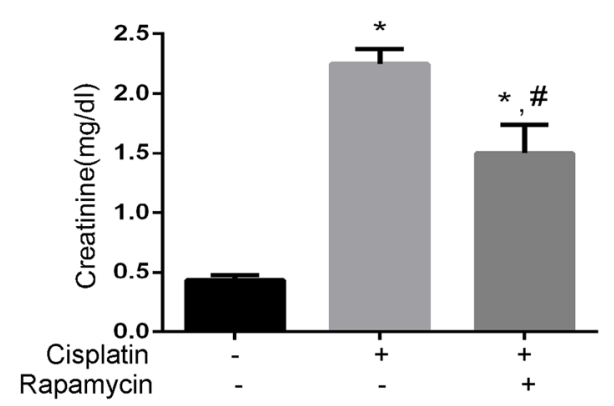

C

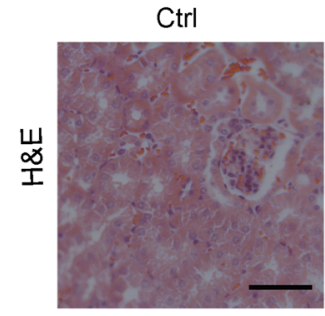

E

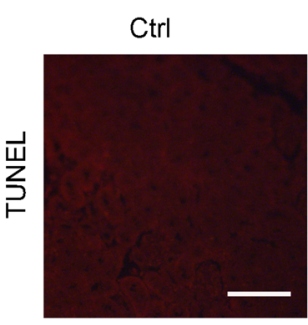

G

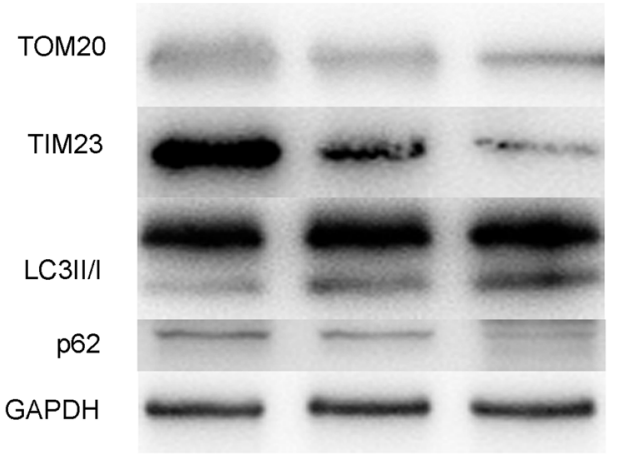

I

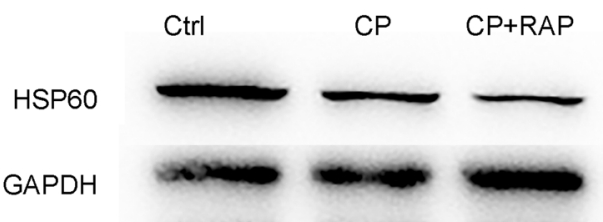

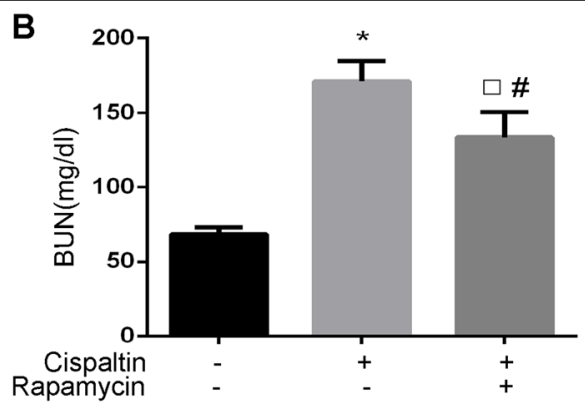

D

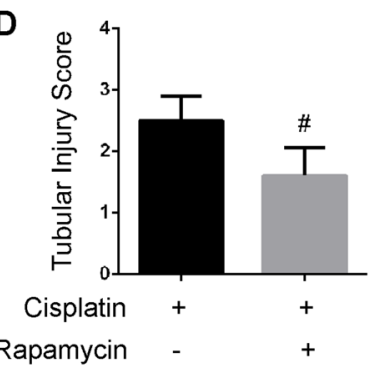

$F$

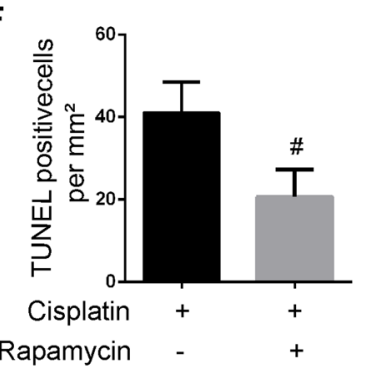

H

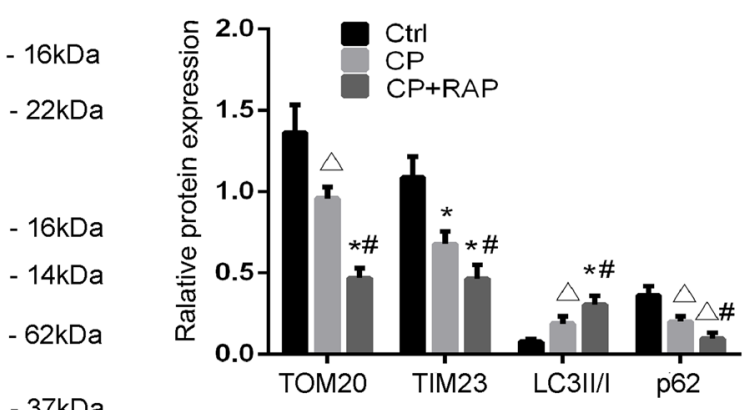

- 37kDa
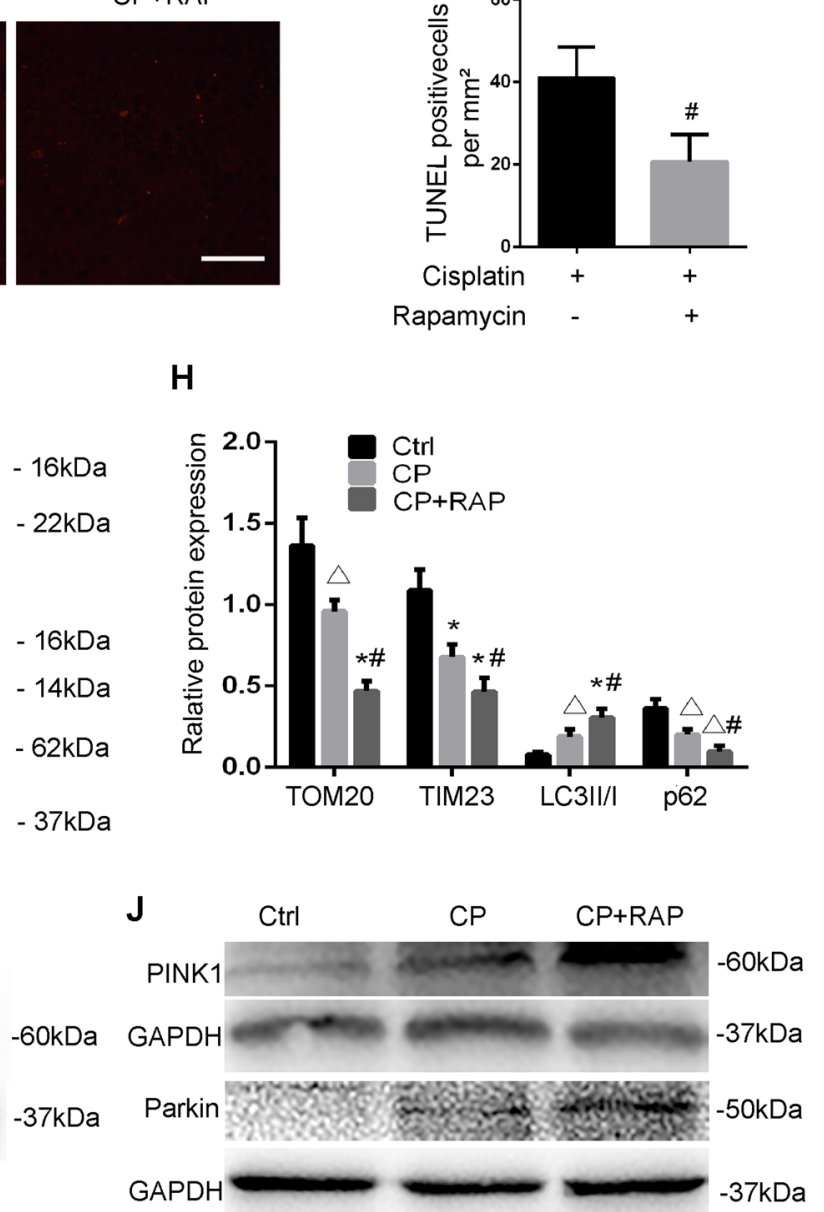

Fig. 3 (See legend on next page.) 
(see figure on previous page)

Fig. 3 Rapamycin enhances mitophagy and protects against cisplatin-induced kidney injury in mice. C57BL/6 mice (male, 8-10 weeks old) were divided into three groups for following treatments, respectively: (1) saline control; (2) cisplatin treatment for 72 h; and (3) cisplatin + rapamycin treatment for $72 \mathrm{~h}$. Cisplatin was injected at $30 \mathrm{mg} / \mathrm{kg}$. For rapamycin, $1 \mathrm{mg} / \mathrm{kg}$ rapamycin was injected $1 \mathrm{~h}$ prior to and 1 day after cisplatin injection. a, b Blood samples were collected for measurements of BUN and serum creatinine. c Representative histology shown by H\&E staining. Tubular injury was indicated by tubular dilation/flattening, loss of brush border, sloughing of cells into tubular lumen, formation of tubular casts, tubular degeneration, and vacuolization. Scale bar, $50 \mu \mathrm{M}$. d Pathological score of tubular damage in cisplatin and cisplatin + rapamycin groups. Tissue injury was scored by the percentage of damaged renal tubules ( 0 , no damage; 1, $<25 \% ; 2,25-50 \% ; 3,50-75 \%$; and 4, $>75 \%$ ). e Representative images of TUNEL staining. Scale bar, $50 \mu \mathrm{M}$. f Quantification of TUNEL-positive cells in cisplatin and cisplatin + rapamycin groups. $\mathbf{g}, \mathbf{i}, \mathbf{j}$ Renal cortex tissues were analyzed for immunoblot analysis of TOM20, TIM23, LC3IIII, p62, HSP60, PINK1, Parkin, and GAPDH. $\mathbf{h}$ Densitometry analysis of proteins signals on immunoblots. The protein signals were divided by GAPDH signal of the same samples to determine the ratios. Data in $\mathbf{a}, \mathbf{b}$ and $\mathbf{d}, \mathbf{f}, \mathbf{h}, \mathbf{j}$ are expressed as mean \pm SD. $n=3,{ }^{*} P<0.001,{ }^{* *} P<0.01,{ }^{* * *} P<0.05$ vs. the control group; ${ }^{\#} P<0.05$ vs. the cisplatin group
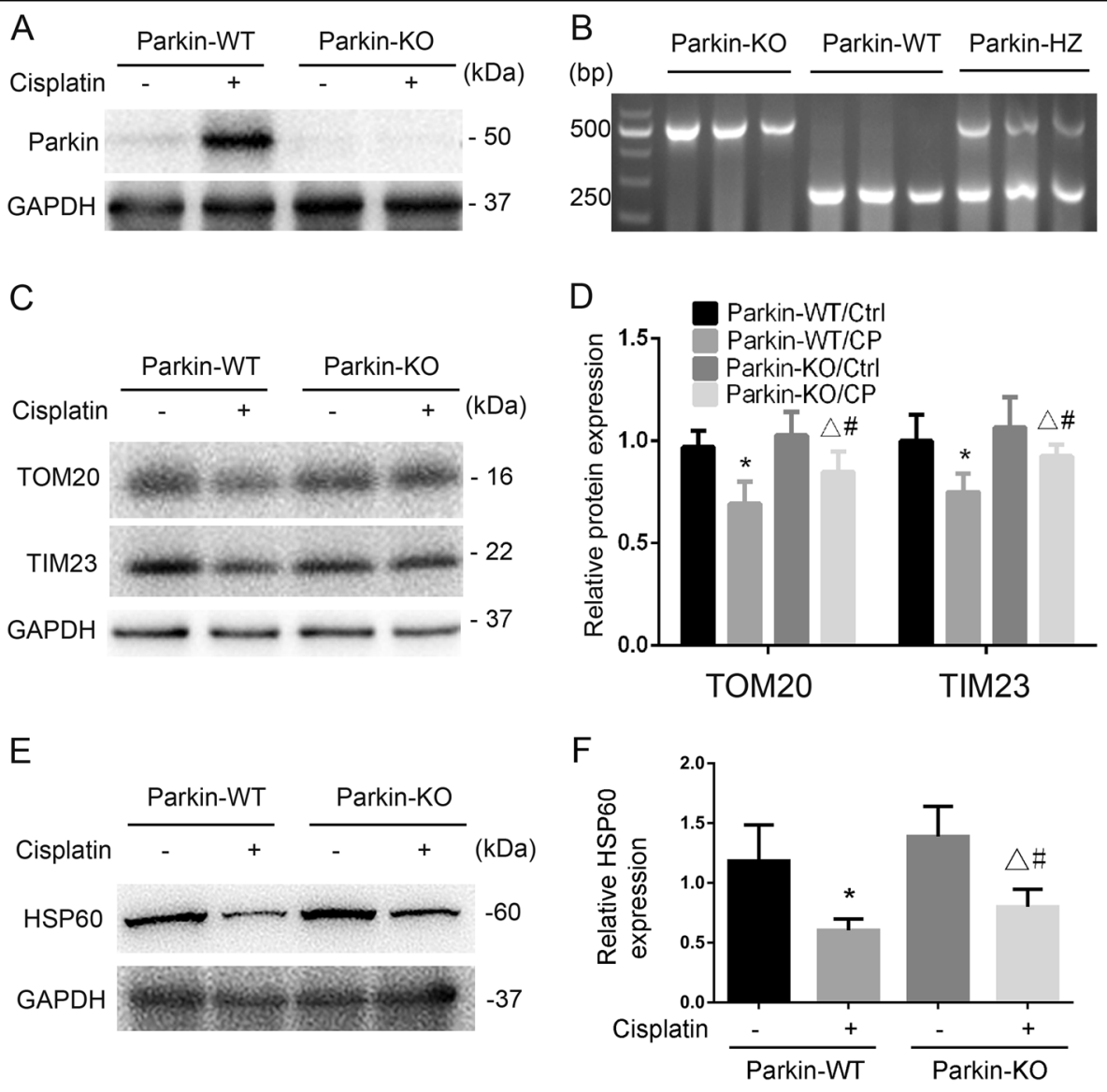

Fig. 4 Mitophagy is suppressed in Parkin-KO mice during cisplatin nephrotoxicity. Whole tissue lysate of kidney cortex was collected from Parkin-KO and wild-type (Parkin-WT) littermate mice. a, c, e Immunoblot analysis of Parkin, PINK1, TOM20, TIM23, HSP60, and GAPDH. b Representative images of PCR-based genotyping. Genomic DNA was extracted from ear biopsy and amplified to detect wild-type (WT), Parkin KO, or Parkin heterozygote $(\mathrm{HZ})$ alleles as indicated. $\mathbf{d}$, $\mathbf{f}$ Densitometry analysis of TOM20, TIM23, and HSP60 signals on immunoblots. Mean \pm SD. $n=4$, ${ }^{*} P<0.001$ vs. the Parkin-WT control group. ${ }^{* *} P<0.05$ vs. the Parkin-KO control group. ${ }^{\#} P<0.05$ vs. Parkin-WT cisplatin group

WT mice, as indicated by tubular dilation, brush border loss, cell lysis, cast formation, and tubular degeneration (Fig. 6c). By quantification, the tubular damage score was 3.4 for Parkin-KO mice and 2.3 for WT (Fig. 6d). Further examination by TUNEL assay also showed more apoptosis in kidney tissues of Parkin-KO mice (Fig. 6e, f). Consistently, cisplatin-induced robust higher levels of caspase-3 activation in Parkin-KO kidney tissues (Fig. 6g, h).

We further examined cisplatin-induced nephrotoxicity in PINK1-KO mice. As shown in Fig. 7a, PINK1-KO mice suffered more severe renal damage than WT mice after $72 \mathrm{~h}$ of cisplatin treatment. PINK1-KO mice had $3.00 \mathrm{mg} /$ dl serum creatinine that was higher than that of WT mice 

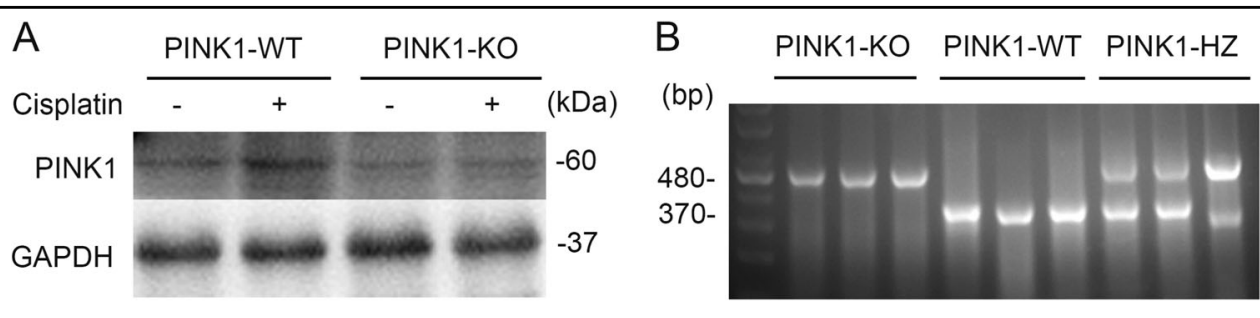

C

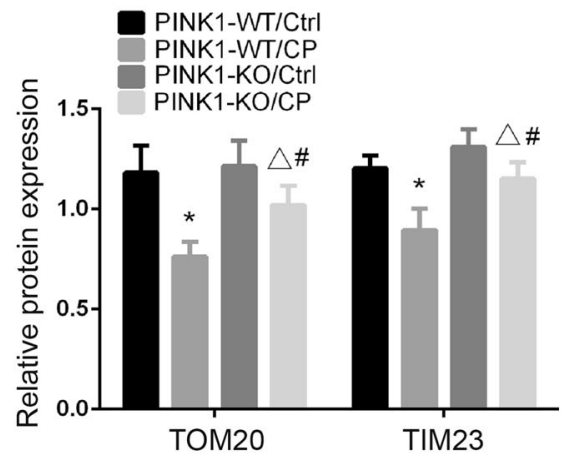

E
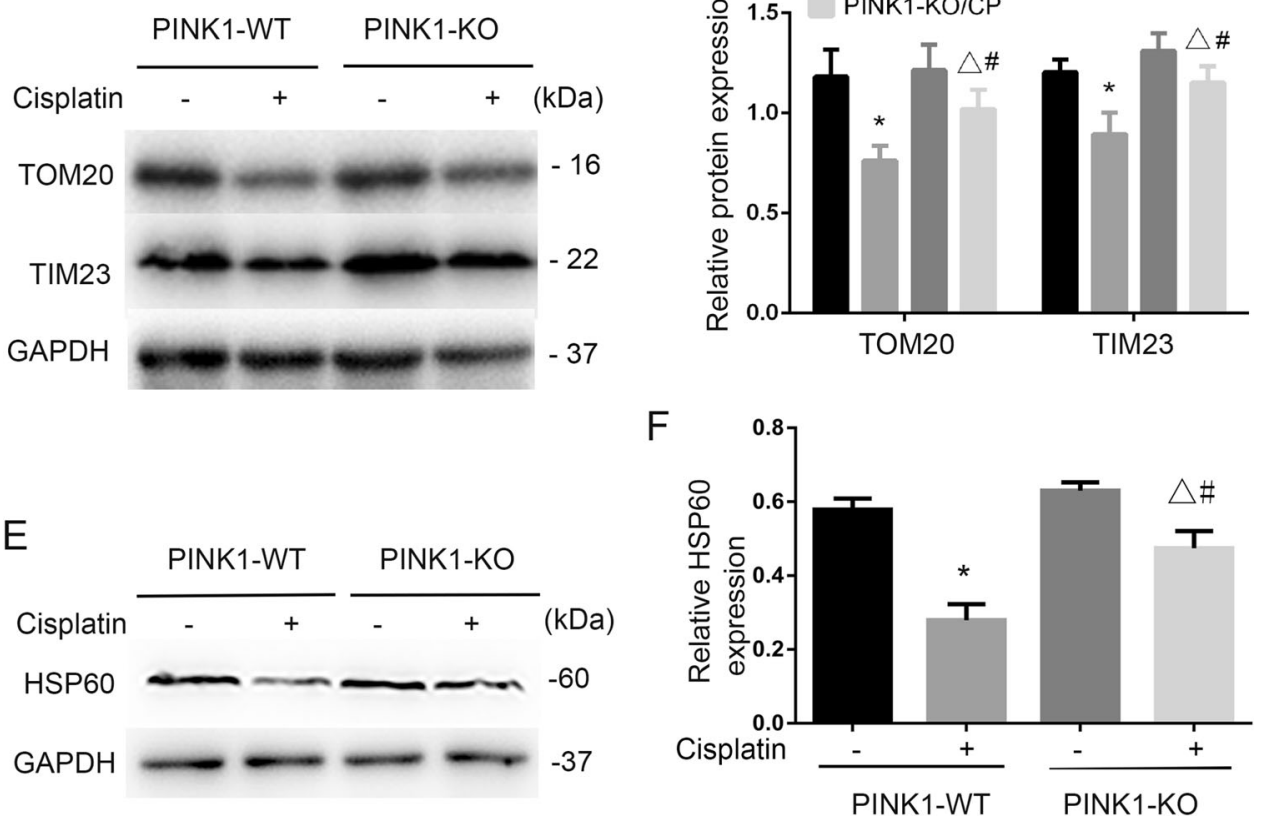

G

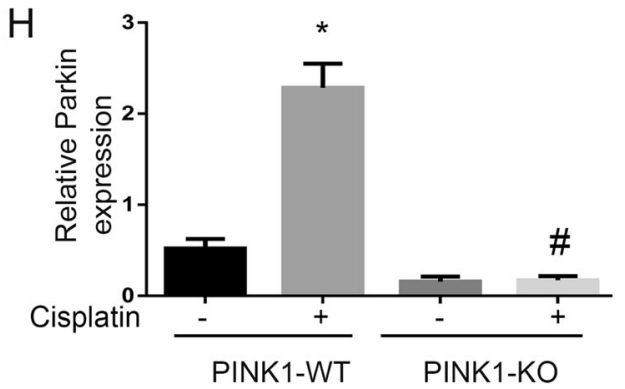

Fig. 5 Mitophagy is inhibited in PINK1-KO mice during cisplatin nephrotoxicity. a, c, e Kidney cortex tissues was collected from PINK1-KO mice and PINK1-WT littermates for immunoblot analysis of Parkin, PINK1, TOM20, TIM23, HSP60, and GAPDH. b Representative images of PCR-based genotyping. Genomic DNA was extracted from ear biopsy and amplified to detect wild-type (WT) and PINK1-KO and PINK1 heterozygote (HZ) allele as indicated. d, f Densitometry of TOM20, TIM23, and HSP60 signals. g, h After cisplatin treatment, renal cortex was collected and extracted mitochondrial (mito) fractions followed by immunoblot analysis of Parkin, COX IV (mitochondrial marker). $\mathbf{g}$ Representative blots. $\mathbf{h}$ Densitometry of Parkin signals. Mean \pm SD. $n=4,{ }^{*} P<0.001$ vs. the PINK1-WT control group. ${ }^{* *} P<0.05$ vs. the Parkin-KO control group. ${ }^{\# P}<0.05$ vs. PINK1-WT cisplatin group

( $2.07 \mathrm{mg} / \mathrm{dl})$. BUN did not significantly differ in these two groups (Fig. 7b), but histological analysis revealed that PINK1-KO mice had more widespread tubular damage as compared with WT mice (Fig. 7c). Renal tubule damage scores were 2.3 for PINK1-KO mice and 3.6 for WT mice, respectively during cisplatin treatment (Fig. $7 \mathrm{~d}$ ). In addition, TUNEL assay demonstrated that there was significant more tubular cell apoptosis after cisplatin in PINK1-KO mice than WT (Fig. 7e, f). Immunoblot analysis also showed a higher induction of cleaved/active caspase 3 in kidney tissue after cisplatin treatment in PINK1-KO mice (Fig. 7g, h). 

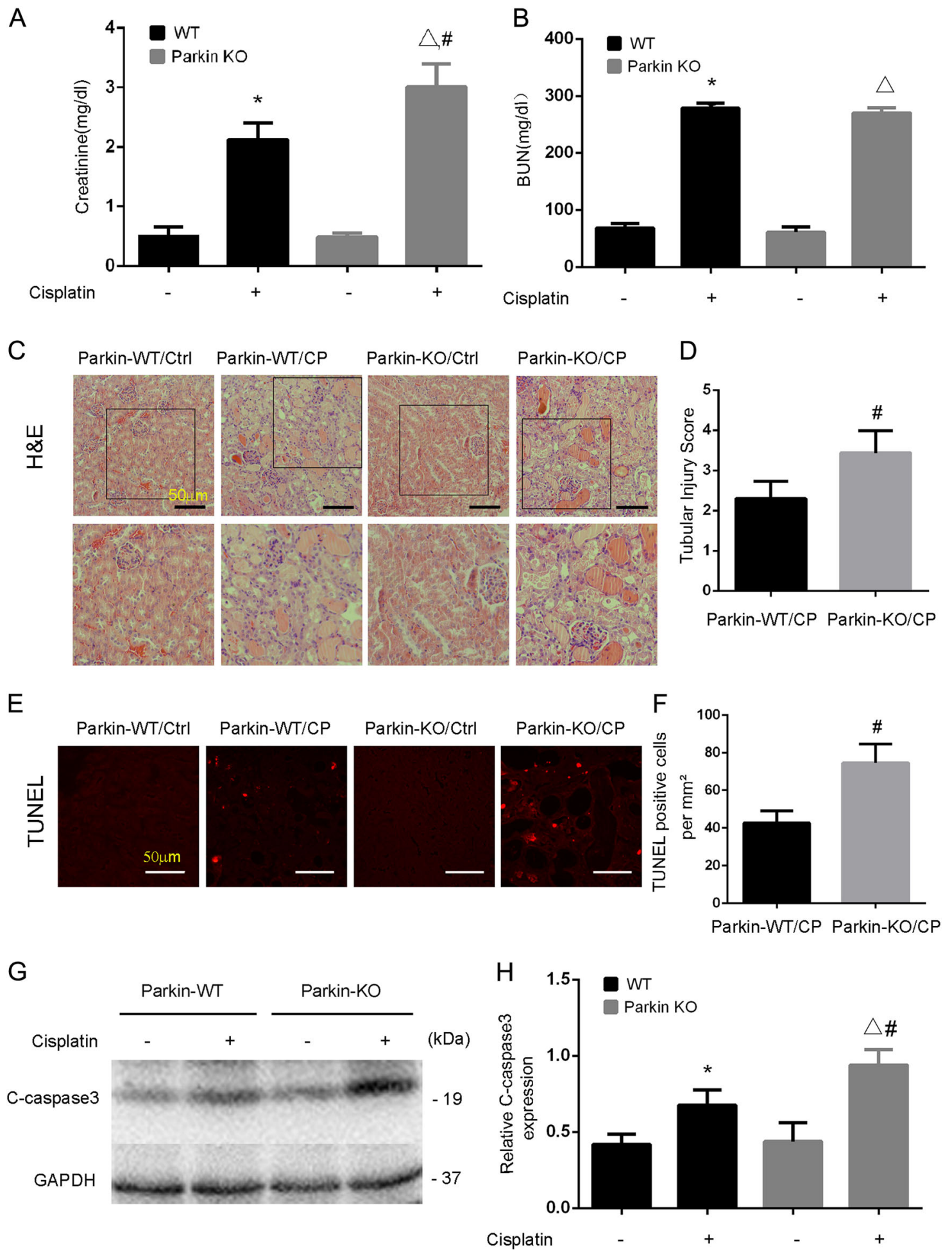

Fig. 6 Deficiency of Parkin aggravates cisplatin-induced kidney injury. Parkin-KO mice and wide-type (WT) mice (male, 8-10 weeks) were injected with $30 \mathrm{mg} / \mathrm{kg}$ cisplatin or saline for $72 \mathrm{~h}$. $\mathbf{a}, \mathbf{b}$ Blood samples were collected for measurements of BUN and serum creatinine. c Representative histology shown by H\&E staining. Tubular injury was indicated by tubular dilation/flattening, loss of brush border, sloughing of cells into tubular lumen, formation of tubular casts, tubular degeneration, and vacuolization. Scale bar, $50 \mu \mathrm{M}$. d Pathological score of tubular damage in cisplatin and cisplatin + rapamycin groups. Tissue injury was scored by the percentage of damaged renal tubules ( 0 , no damage; $1,<25 \% ; 2,25-50 \% ; 3,50-75 \%$; and 4 , >75\%). e Representative images of TUNEL staining. Scale bar, $50 \mu \mathrm{M}$. $\mathbf{f}$ Quantification of TUNEL-positive cells in cisplatin treatment for the wild-type groups and Parkin-KO groups. g Immunoblot analysis for cleaved caspase-3 (C-caspase 3). $\mathbf{h}$ Densitometry analysis of the cleaved-caspase 3 signals. Mean \pm SD. $n=4,{ }^{*} P<0.001$ vs. the Parkin-WT control group. ${ }^{* *} P<0.001$ vs. the Parkin-KO control group. ${ }^{*} P<0.05$ vs. Parkin-WT cisplatin group 

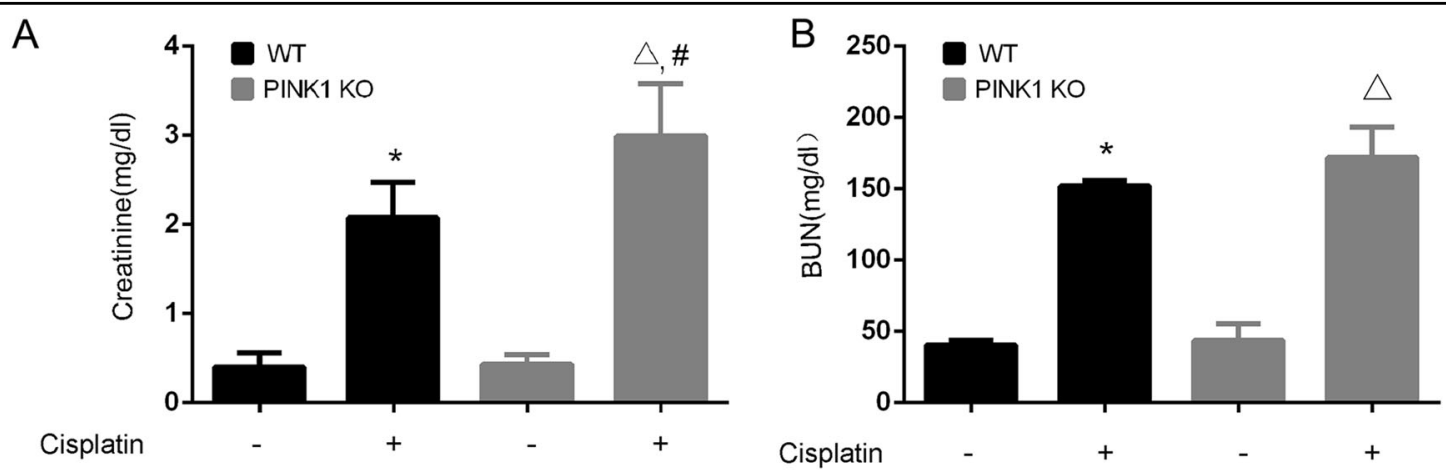

C

PINK1-WT/Ctrl PINK1-WT/CP

PINK1-KO/CtrI

$\mathrm{PINK} 1-\mathrm{KO} / \mathrm{CP}$

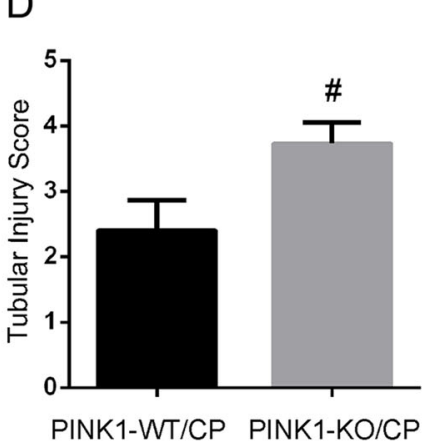

E

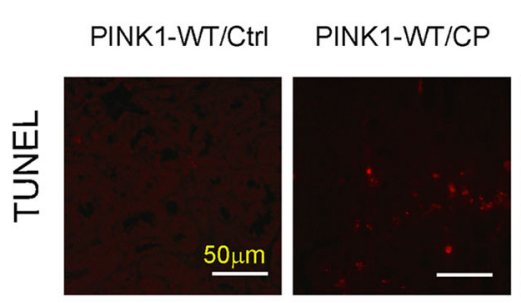

G

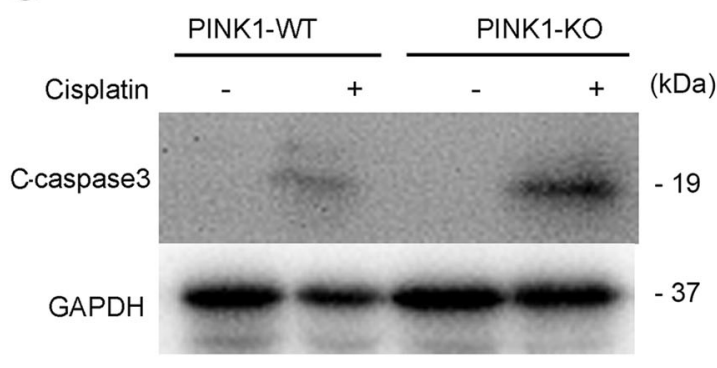

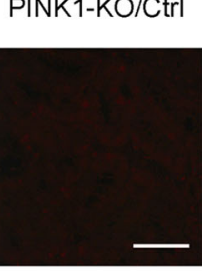

$\mathrm{PINK} 1-\mathrm{KO} / \mathrm{CP}$

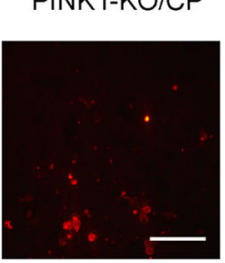

$\mathrm{F}$

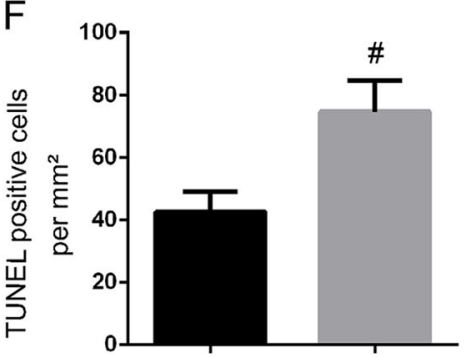

PINK1-WT/CP PINK1-KO/CP

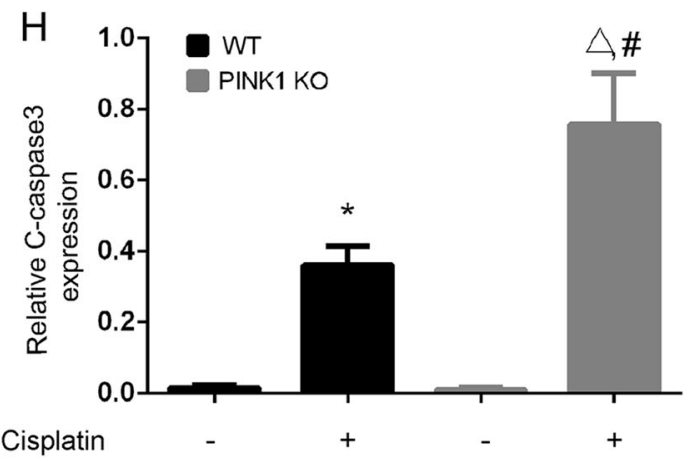

Fig. 7 PINK1 deficiency worsens cisplatin-induced kidney injury. PINK1-KO mice and their wild-type (WT) mice (male, 8-10 weeks) were injected with $30 \mathrm{mg} / \mathrm{kg}$ cisplatin/or saline for $72 \mathrm{~h}$. a, b Blood samples were collected for measurement of BUN and serum creatinine. c, e Kidney tissues were harvested for H\&E staining of histology (c) and TUNEL assay of apoptosis (e). Scale bar, $50 \mu \mathrm{M}$. In H\&E staining, yubular injury was indicated by tubular dilation/flattening, loss of brush border, sloughing of cells into tubular lumen, formation of tubular casts, tubular degeneration, and vacuolization. Scale bar, $50 \mu \mathrm{M}$. d Pathological score of tubular damage. Tissue injury was scored by the percentage of damaged renal tubules $(0$, no damage; 1, <25\%; 2, 25-50\%; 3, 50-75\%; 4, >75\%). f Quantification of TUNEL-positive cells in kidney tissues. $\mathbf{g}$ Immunoblot analysis for cleaved caspase 3. h Densitometry analysis of the cleaved caspase 3 signals. Mean \pm SD. $n=4,{ }^{*} P<0.001$ vs. PINK1-WT control group. ${ }^{* *} P<0.001$ vs. Parkin-KO control group. ${ }^{\#} P<0.05$ vs. PINK1-WT cisplatin group 
These results indicate that cisplatin-induced nephrotoxicity is aggravated in Parkin- or PINK1-KO mice, supporting a protective role of PINK1/Parkin-mediated mitophagy in cisplatin nephrotoxicity.

\section{Discussion}

Autophagy is activated for kidney protection in cisplatin nephrotoxicity ${ }^{28-32}$. However, the roles and mechanisms of mitophagy underlying cisplatin nephrotoxicity remain largely unclear. In this study, we analyzed the potential pathologic effects of mitophagy in cisplatin nephrotoxicity using both cell and animal models. Notably, this study has tested both pharmacologic modulators and genetic $\mathrm{KO}$ models to determine the role of mitophagy. Our results provide evidence for the activation of mitophagy in kidney tissues following cisplatin treatment. Rapamycin augmented mitophagy and afforded protection against nephrotoxic kidney injury. Notably, KO of PINK1- or Parkin-suppressed mitophagy in kidneys of cisplatintreated mice, suggesting that PINK1/Parkin mediates the main pathway of mitophagy in nephrotoxicity. Importantly, PINK1 and Parkin KO mice showed more severe kidney injury following cisplatin treatment, supporting a protective role of PINK1/Parkin-mediated mitophagy in cisplatin nephrotoxicity.

A recent study by Yuan's laboratory ${ }^{27}$ showed that in a human kidney proximal tubular HK2 cell line, knockdown of PINK1 or Parkin led to a decrease of mitophagy during cisplatin treatment, which was associated with an increase in cell injury. In contrast, overexpression of these two genes enhanced mitophagy and protected HK2 cells against cisplatin-induced cell death, providing the first evidence for a protective role of PINK1/Parkin-mediated mitophagy in cisplatin nephrotoxicity. However, it remained elusive if the PINK1/Parkin pathway was activated by cisplatin in vivo in kidneys, how much it contributed to mitophagy in cisplatin nephrotoxicity, and what role it might play in this disease condition. Our present study has addressed these important questions by using mouse models. By analyzing mitochondrial proteins and DNA, we have provided evidence of mitophagy activation in kidney tissues during cisplatin treatment (Figs. 1, 2). Pharmacological activation of autophagy by rapamycin enhanced mitophagy (Fig. 3) and protected against kidney injury. It is noteworthy that rapamycin increases autophagy in general so its effect includes, but is not limited to, mitophagy. Thus, the protective effect of rapamycin observed in our study was not solely due to mitophagy activation. Our further work showed that KO of either PINK1 or Parkin reduced mitophagy, indicating that the PINK1/Parkin pathway is the main pathway of mitophagy during cisplatin nephrotoxicity. Remarkably, KO of either PINK1 or Parkin was associated with significantly higher cisplatin nephrotoxicity in mice
(Figs. 6, 7), supporting a protective role of PINK1/Parkinmediated mitophagy in this disease condition.

In both ischemic and cisplatin nephrotoxic kidney injury, mitochondria become fragmented, contributing to renal tubular damage ${ }^{33}$. Mitochondrial fragmentation under these conditions is caused by the loss of mitochondrial dynamics, specifically acceleration of mitochondrial fission and arrest of mitochondrial fusion ${ }^{10,34}$. An important question in this research area is the fate of the fragmented mitochondria. By our observation, some fragmented mitochondria may refuse into long filamentous mitochondria when the insult is removed, whereas others may become irreversibly damaged, for example, by the "attacking" or insertion of Bax into mitochondrial outer membrane ${ }^{35}$. Our present data suggest yet another outcome, i.e., clearance of fragmented mitochondria by mitophagy. As a matter of fact, for mitophagy to occur, mitochondria need to be fragmented. In this regard, Yuan's laboratory demonstrated that Drp-1-dependent mitochondrial fission is required for mitophagy during cisplatin treatment of HK2 cells ${ }^{36}$. Consistently, the latest work by Li et al. ${ }^{37}$ showed that mitophagy during renal ischemia-reperfusion injury depends on Drp-1mediated mitochondrial fragmentation.

Thus far, very few studies examined mitophagy in experimental models of kidney injury. Ishihara et al. ${ }^{38}$ reported that Sestrin-2 and BNIP3 might regulate mitophagy in renal tubular cells under conditions of oxidative stress and hypoxia, respectively. Our recent study ${ }^{26}$ demonstrated a protective role of the PINK1/Parkin pathway of mitophagy in renal ischemia-reperfusion injury by using gene $\mathrm{KO}$ models. In nephrotoxic models, our current study and the work by Yuan's laboratory ${ }^{27,36}$ also support a protective role of PINK1/Parkinmediated mitophagy in cisplatin-induced kidney injury. Together, these studies suggest that multiple pathways of mitophagy may be activated in renal tubular cells in pathological conditions and, among them, the PINK1/Parkin pathway is a main pathway for kidney protection.

Mitophagy involves adapter or receptor proteins. In this regard, Geisler et al. ${ }^{20}$ reported p62 as the main receptor protein for Pink1/Parkin-mediated mitophagy. However, later studies identified several other proteins as mitophagy receptors, including TAX1BP1, NDP52, NBR1, p62, and OPTN. In 2015, Lazarou et al. ${ }^{39}$ conducted a comprehensive study of these known adapters and suggested that OPTN and NDP52 as the most important adapters for Pink1/Parkin-dependent mitophagy. Future research should investigate the receptor(s) for mitophagy in cisplatin-induced kidney injury and nephrotoxicity.

How does mitophagy protect kidney cells and tissues? It is generally understood that mitophagy, as a 
selective form of autophagy, is used by cells to degrade dysfunctional or damaged mitochondria. Cisplatin nephrotoxicity is associated with mitochondrial damage, especially that occurring in kidney tubular cells ${ }^{8}$. Mitochondrial damage may lead to cell death passively due to the loss of ATP production. On the other hand, it may also actively promote cell death. For example, damaged mitochondria may release apoptosis-activating factors, such as cytochrome c. In addition, damaged mitochondria may produce excessive amount of reactive oxygen species that are toxic to cells. By removing damaged mitochondria, mitophagy may reduce the level of cell stress and promote cell survival during cisplatin nephrotoxicity.

In summary, our study has demonstrated that mitophagy is activated in both cell and animal models of cisplatin nephrotoxicity. Mechanistically, mitophagy induction under this condition is mediated mainly by the PINK1/Parkin pathway. Upon activation, mitophagy protects kidneys against cisplatin toxicity likely by removing damaged mitochondria in renal tubular cells to promote cell survival.

\section{Methods and materials}

\section{Cells, antibodies, and special reagents}

The Boston University mouse proximal tubular (BUMPT) cell line (BUMPT-306) was initially obtained from Drs. William Lieberthal and John Shwartz at Boston University ${ }^{40}$. The sources of the primary antibodies in this study were as follows: anti-LC3 (12741S), anti-TOM20 (42406S), anti-Parkin (4211S), anti-GAPDH (5174S), anticleaved caspase-3 (9664S), HSP60 (12165P), and COX IV (4850P) from Cell Signaling Technologies; anti-TIM23 (11123-1-AP) and anti-p62 (18420-1-AP) from Proteintech; and anti-PINK1 (P0076) from Sigma-Aldrich. All secondary antibodies were from Thermo Fisher Scientific. Other reagents were purchased from SigmaAldrich, such as cisplatin and rapamycin. Both BUN and serum creatinine measurement kits were from BioAssay Systems.

\section{Animals}

PINK1-KO mice and Parkin-KO mice were originally obtained from the Institute of Precision Medicine of Xiangya Hospital at Central South University (Changsha, Hunan, China), and these mouse lines were previously described $^{41,42}$. C57BL/6 mice (8-10 weeks old, male) were purchased from SJA Laboratory Animal Corporation (Changsha, Hunan, China). Male mice of 8-12 weeks age were used in this study. All animals were housed in a pathogen-free animal facility under 12 -h light/12-h dark pattern with free access to water and food. All experiments were executed in line with the protocol approved by the Institutional Animal Care and Use Committee of the Second Xiangya Hospital of Central South University.

\section{Cisplatin treatment of mice}

Mice were injected intraperitionally with $30 \mathrm{mg} / \mathrm{kg}$ body weight cisplatin, and control mice received an intraperitional injection of the equal volume of $0.9 \%$ saline. Blood was collected for BUN and serum creatinine measurement at time $0,24,48$, and $72 \mathrm{~h}$ after cisplatin treatment. Most mice were sacrificed at $72 \mathrm{~h}$ after cisplatin injection to collect blood samples for serum creatinine and BUN measurement, and collect renal tissues for histological and immunoblot analysis.

\section{Rapamycin treatment of mice}

Totally, $1 \mathrm{mg} / \mathrm{kg}$ rapamycin or equal volume of $0.9 \%$ saline was injected $1 \mathrm{~h}$ prior to and $24 \mathrm{~h}$ after cisplatin $(30 \mathrm{mg} / \mathrm{kg})$ and then sacrificed at $72 \mathrm{~h}$. The mice were divided into three groups for following treatment: (1) control group (injection of the equal volume $0.9 \%$ saline), (2) saline + cisplatin (injection of saline and cisplatin), and (3) rapamycin + cisplatin (injection of rapamycin and cisplatin). The method of blood harvest and kidney collection was the same as described above.

\section{Analysis of renal function}

Renal failure or loss of renal function was revealed by serum creatinine and BUN using commercial kits as previously described ${ }^{43,44}$. After coagulation, blood samples were centrifuged at room temperature to collect the serum. For BUN measurement, serum samples were added to the reaction solution at room temperature for $20 \mathrm{~min}$. The absorbance of $520 \mathrm{~nm}$ was recorded to calculate the value of BUN. For serum creatinine measurement, serum samples were added to reaction solution and the absorbance at $510 \mathrm{~nm}$ was recorded at 0 and $5 \mathrm{~min}$ of reaction. Then BUN and serum creatinine levels were calculated based on standard curves and shown as $\mathrm{mg} / \mathrm{dl}$.

\section{Renal histology}

For histology, kidney tissues were fixed in $4 \%$ paraformaldehyde for paraffin embedding and each sample was sectioned at $4 \mu \mathrm{M}$ for hematoxylin-eosin (H\&E) staining. Histological damage was indicated by tubular dilation/flattening, loss of brush border, sloughing of cells into tubular lumen, formation of tubular casts, tubular degeneration, and vacuolization. Tissue injury was scored by the percentage of damaged renal tubules ( 0 , no damage; $1,<25 \% ; 2,25-50 \%$; 3 , $50-75 \%$; and $4,>75 \%$.) 


\section{Analysis of apoptosis}

Apoptosis were determined in kidney tissues by TUNEL assay by using the In Situ Cell Death Detection Kit from Roche Applied Science (Indianapolis, IN) as described in our recent work ${ }^{43,44}$. Briefly, kidney tissues were embedded in paraffin and deparaffinized with a standard protocol. Tissue kidney sections were permeabilized with 0.1 $\mathrm{M}$ sodium citrate, $\mathrm{pH} 6.0$ at $65^{\circ} \mathrm{C}$ for $30 \mathrm{~min}$ and then incubated with a TUNEL reaction buffer for $1 \mathrm{~h}$ at $37^{\circ} \mathrm{C}$ in a humidified-dark chamber. Positive staining was identified by fluorescence microscopy. Each section was selected for ten representative fields randomly and the TUNEL-positive cells per $\mathrm{mm}^{2}$ was counted. Apoptosis in cultured proximal tubular cells were morphological analyzed after staining with Hoechst33342. The percentage of apoptosis was evaluated according to the cells showing morphological traits including nuclear condensation and fragmentation, and formation of apoptotic bodies. Besides, apoptosis was also assessed by immunoblot analysis of cleaved caspase- 3 .

\section{Quantification of mitochondrial DNA content}

Total cellular DNA of renal cortex tissue was extracted using the DNeasy Blood and Tissue kit (Qiagen, 69506) according to the manufacturer's instruction. The relative content of mtDNA was measured as previously described $^{45,46}$. Briefly, both mtDNA (measured by mitochondrial $16 \mathrm{~S}$ rRNA gene) and nDNA (measured by $\beta 2-$ microglobulin gene) in the same samples were quantified by quantitative polymerase chain reaction (PCR) using a CFX96 Real-Time PCR Detection System (BiO-RAD) with the SyBR GreenER qPCR SuperMix (Invitrogen). The primers were: $\mathrm{mt} 16 \mathrm{~S}$ forward, 5'-ATTCCAA TTCTCCAGGCATACG-3';

mt16S reverse, 5'-GGGGTTCTTGTTTGCCGAGT T-3';

$\beta 2$-microglobulin forward, 5'-AGGGTGTGCAGAATG GGATG-3';

$\beta 2$-microglobulin reverse, 5 -GCTTCCCCCAAAGTCT ACCC-3';

GAPDH forward, 5'-AGGTCGGTGAACGGATTG-3'; GAPDH reverse, 5'-TGTAGACCATGTAGTTGAGG TCA-3'.

\section{Co-localization of autophagosomes with mitochondria}

BUMPT cells were transiently transfected with the LC3GFP fusion plasmid (Addgene) by using Lipofectamine 2000 (Invitrogen) to reveal punctate autophagosome forms upon stimulation. Mitochondria in these cells were labled with MitoTracker Red FM (Molecular Probes, M22425) following the manufacturer's instructions. The cells were examined with an Olympus FV 1000 laserscanning confocal microscope (Olympus Corporation, Tokyo, Japan).

\section{Mitochondrial isolation}

Mitochondria were isolated from kidneys by using mitochondrial extraction kit (Solarbio). Briefly, kidneys were rinsed in cold PBS and homogenized in ice-cold lysis buffer with a Dounce-type glass homogenizer. The homogenate was then centrifuged for three times $(1000 \times g$ for $10 \mathrm{~min}$ at $4{ }^{\circ} \mathrm{C}$ ) to pellet tissue debris and nuclei, yielding a supernatant enriched in mitochondria. Mitochondria from the supernatant were finally pelleted by centrifugation at $12,000 \times \mathrm{g}$ and $4{ }^{\circ} \mathrm{C}$ for $10 \mathrm{~min}$.

\section{Immunoblot analysis}

Immunoblot analysis was conducted by a standard protocol $^{47,48}$.In short, kidney cortex tissue and cell lysates were treated with $2 \%$ sodium dodecyl sulfate (SDS) buffer containing protease inhibitor cocktail (Sigma-Aldrich). Protein concentration was determined with the bicinchoninic acid reagent from Thermo Fisher Scientific. The same amounts $(100 \mu \mathrm{g})$ of protein were loaded in each lane and resolved on SDS-polyacrylamide electrophoresis gel and then transferred to polyvinylidene diflouride membrane which was then blocked with $5 \%$ fat-free milk for $1 \mathrm{~h}$ before incubation with primary antibodies and horseradish peroxidaseconjugated secondary antibodies. The protein bands were revealed with a chemiluminescence kit (Termo Fisher Scientific). Densitometry of protein band signal was analyzed with Image J software (NIH) for quantification.

\section{Statistical analysis}

Qualitative data shown in this study, including immunoblot and cell and tissue images, are representative of at least three separate experiments. Quantitative data are expressed as mean $\pm \mathrm{SD}$. T-test was used to determine the statistical significance between two groups. ANOVA followed by Tukey's post-tests was conducted to identify the statistical significance in the differences among multiple groups. Statistical analysis was implemented by the GraphPad Prism software. $P<0.05$ was considered to indicate significant differences.

\section{Acknowledgments \\ The work was supported partly by the grants from National Natural Science Foundation of China (81720108008 and 81430017), the National Institutes of Health of USA, and Department of Veterans Administration of USA. \\ Author details \\ 'Department of Nephrology, The Second Xiangya Hospital at Central South University, Changsha, Hunan, China. ${ }^{2}$ Department of Emergency Medicine, The Second Xiangya Hospital at Central South University, Changsha, Hunan, China. ${ }^{3}$ Institute of Precision Medicine, Xiangya Hospital at Central South University, Changsha, Hunan, China. ${ }^{4}$ Department of Cellular Biology and Anatomy, Medical College of Georgia at Augusta University and Charlie Norwood VA Medical Center, Augusta, GA, USA}

Conflict of interest

The authors declare that they have no conflict of interest. 


\section{Publisher's note}

Springer Nature remains neutral with regard to jurisdictional claims in published maps and institutional affiliations.

Received: 11 June 2018 Revised: 12 October 2018 Accepted: 16 October 2018

Published online: 01 November 2018

\section{References}

1. Wang, D. \& Lippard, S. J. Cellular processing of platinum anticancer drugs. Nat. Rev. Drug Discov. 4, 307-320 (2005).

2. Rancoule, C. et al. 50th anniversary of cisplatin. Bull. Cancer 104, 167-176 (2017).

3. Miller, R. P., Tadagavadi, R. K, Ramesh, G. \& Reeves, W. B. Mechanisms of cisplatin nephrotoxicity. Toxins (Basel) 2, (2490-2518 (2010).

4. Pabla, N. et al. Inhibition of PKCdelta reduces cisplatin-induced nephrotoxicity without blocking chemotherapeutic efficacy in mouse models of cancer. J. Clin. Invest. 121, 2709-2722 (2011).

5. Ozkok, A. \& Edelstein, C. L. Pathophysiology of cisplatin-induced acute kidney injury. Biomed. Res. Int. 2014, 967826 (2014).

6. dos Santos, N. A., Carvalho Rodrigues, M. A., Martins, N. M. \& dos Santos, A. C. Cisplatin-induced nephrotoxicity and targets of nephroprotection: an update. Arch. Toxicol. 86, 1233-1250 (2012).

7. Zhu, S., Pabla, N., Tang, C., He, L. \& Dong, Z. DNA damage response in cisplatininduced nephrotoxicity. Arch. Toxicol. 89, 2197-2205 (2015).

8. Yang, Y., Liu, H., Liu, F. \& Dong, Z. Mitochondrial dysregulation and protection in cisplatin nephrotoxicity. Arch. Toxicol. 88, 1249-1256 (2014).

9. Price, P. M., Safirstein, R. L. \& Megyesi, J. The cell cycle and acute kidney injury. Kidney Int. 76, 604-613 (2009).

10. Zhan, M., Brooks, C., Liu, F., Sun, L. \& Dong, Z. Mitochondrial dynamics: regulatory mechanisms and emerging role in renal pathophysiology. Kidney Int. 83, 568-581 (2013).

11. Parikh, S. M. et al. Mitochondrial function and disturbances in the septic kidney. Semin. Nephrol. 35, 108-119 (2015).

12. Agarwal, A. et al. Cellular and molecular mechanisms of AKl. J. Am. Soc. Nephrol. 27, 1288-1299 (2016).

13. Bhargava, P. \& Schnellmann, R. G. Mitochondrial energetics in the kidney. Nat. Rev. Nephrol. 13, 629-646 (2017).

14. Szeto, H. H. Pharmacologic approaches to improve mitochondrial function in AKI and CKD. J. Am. Soc. Nephrol. 28, 2856-2865 (2017).

15. Ding, W. X. \& Yin, X. M. Mitophagy: mechanisms, pathophysiological roles, and analysis. Biol. Chem. 393, 547-564 (2012).

16. Tang, C., He, L., Liu, J. \& Dong, Z. Mitophagy: basic mechanism and potential role in kidney diseases. Kidney Dis. 1, 71-79 (2015).

17. Youle, R. J. \& Narendra, D. P. Mechanisms of mitophagy. Nat. Rev. Mol. Cell Biol. 12, 9-14 (2011).

18. Matsuda, N. et al. PINK1 stabilized by mitochondrial depolarization recruits Parkin to damaged mitochondria and activates latent Parkin for mitophagy. J. Cell Biol. 189, 211-221 (2010).

19. Kane, L. A. et al. PINK1 phosphorylates ubiquitin to activate Parkin E3 ubiquitin ligase activity. J. Cell Biol. 205, 143-153 (2014).

20. Geisler, S. et al. PINK1/Parkin-mediated mitophagy is dependent on VDAC1 and p62/SQSTM1. Nat. Cell Biol. 12, 119-131 (2010).

21. Gegg, M. E. et al. Mitofusin 1 and mitofusin 2 are ubiquitinated in a PINK1/ parkin-dependent manner upon induction of mitophagy. Hum. Mol. Genet. 19, 4861-4870 (2010).

22. Shi, J. et al. NBR1 is dispensable for PARK2-mediated mitophagy regardless of the presence or absence of SQSTM1. Cell Death Dis. 6, e1943 (2015).

23. Keane, P. C., Kurzawa, M., Blain, P. G. \& Morris, C. M. Mitochondrial dysfunction in Parkinson's disease. Parkinsons. Dis. 2011, 716871 (2011).

24. Mohan, D., Balasubramanian, E. D., Ravindran, S. \& Kurian, G. A. Renal mitochondria can withstand hypoxic/ischemic injury secondary to renal failure in uremic rats pretreated with sodium thiosulfate. Indian J. Pharmacol. 49, 317-321 (2017).
25. Flemming, N. B., Gallo, L. A. \& Forbes, J. M. Mitochondrial dysfunction and signaling in diabetic kidney disease: oxidative stress and beyond. Semin. Nephrol. 38, 101-110 (2018).

26. Tang $C_{\text {., }}$ et al. PINK1-PRKN/PARK2 pathway of mitophagy is activated to protect against Renal ischemia-reperfusion injury. Autophagy 14, 880-897 (2018).

27. Zhao, C. et al. Pink1/Parkin-mediated mitophagy play a protective role in cisplatin induced renal tubular epithelial cells injury. Exp. Cell Res. 350, 390-397 (2017).

28. Jiang, M. et al. Autophagy in proximal tubules protects against acute kidney injury. Kidney Int. 82, 1271-1283 (2012).

29. Periyasamy-Thandavan, S. et al. Autophagy is cytoprotective during cisplatin injury of renal proximal tubular cells. Kidney Int. 74, 631-640 (2008).

30. Takahashi, A. et al. Autophagy guards against cisplatin-induced acute kidney injury. Am. J. Pathol. 180, 517-525 (2012).

31. Zhang, D. et al. Protein kinase Cdelta suppresses autophagy to induce kidney cell apoptosis in cisplatin nephrotoxicity. J. Am. Soc. Nephrol. 28, 1131-1144 (2017).

32. Yang, C., Kaushal, V., Shah, S. V. \& Kaushal, G. P. Autophagy is associated with apoptosis in cisplatin injury to renal tubular epithelial cells. Am. J. Physiol. Ren. Physiol. 294, F777-F787 (2008).

33. Brooks, C., Cho, S. G., Wang, C. Y., Yang, T. \& Dong, Z. Fragmented mitochondria are sensitized to Bax insertion and activation during apoptosis. Am. J. Physiol. Cell. Physiol. 300, C447-C455 (2011).

34. Brooks, C. et al. Bak regulates mitochondrial morphology and pathology during apoptosis by interacting with mitofusins. Proc. Natl Acad. Sci. USA 104, 11649-11654 (2007).

35. Brooks, C. \& Dong, Z. Regulation of mitochondrial morphological dynamics during apoptosis by Bcl-2 family proteins: a key in Bak? Cell Cycle 6, 3043-3047 (2007).

36. Zhao, C. et al. Drp1-dependent mitophagy protects against cisplatininduced apoptosis of renal tubular epithelial cells by improving mitochondrial function. Oncotarget 8, 20988-21000 (2017).

37. Li, N., Wang, H., Jiang, C. \& Zhang, M. Renal ischemia/reperfusion-induced mitophagy protects against renal dysfunction via Drp1-dependent-pathway. Exp. Cell Res. 369, 27-33 (2018).

38. Ishihara, M. et al. Sestrin-2 and BNIP3 regulate autophagy and mitophagy in renal tubular cells in acute kidney injury. Am. J. Physiol. Ren. Physiol. 305, F495-F509 (2013).

39. Lazarou, M. et al. The ubiquitin kinase PINK1 recruits autophagy receptors to induce mitophagy. Nature 524, 309-314 (2015).

40. Bhatt, K. et al. MicroRNA-34a is induced via p53 during cisplatin nephrotoxicity and contributes to cell survival. Mol. Med. 16, 409-416 (2010).

41. Xiong, $\mathrm{H}$. et al. Parkin, PINK1, and DJ-1 form a ubiquitin E3 ligase complex promoting unfolded protein degradation. J. Clin. Invest. 119, 650-660 (2009).

42. Choo, Y. S., Tang, C. \& Zhang, Z. Critical role of PINK1 in regulating Parkin protein levels in vivo. Arch. Neurol. 68, 684-685 (2011).

43. Brooks, C., Wei, Q., Cho, S. G. \& Dong, Z. Regulation of mitochondrial dynamics in acute kidney injury in cell culture and rodent models. J. Clin. Invest. 119, 1275-1285 (2009).

44. Guo, C. et al. DNA methylation protects against cisplatin-induced kidney injury by regulating specific genes, including interferon regulatory factor 8 . Kidney Int. 92, 1194-1205 (2017).

45. Bueno, M. et al. PINK1 deficiency impairs mitochondrial homeostasis and promotes lung fibrosis. J. Clin. Invest. 125, 521-538 (2015).

46. Venegas, V., Wang, J., Dimmock, D. \& Wong, L. J. Real-time quantitative PCR analysis of mitochondrial DNA content. Curr. Protoc. Hum. Genet. Unit 19, 17 (2011). Chapter 19.

47. Hao, J. et al. Induction of microRNA-17-5p by p53 protects against renal ischemia-reperfusion injury by targeting death receptor 6. Kidney Int. 91, 106-118 (2017).

48. Liu, J. et al. Histone deacetylase inhibitors protect against cisplatin-induced acute kidney injury by activating autophagy in proximal tubular cells. Cell Death Dis. 9, 322 (2018). 